\section{Diagnóstico de la estrategia editorial de medios informativos chilenos en Twitter mediante un clasificador de noticias automatizado*}

Diagnosing editorial strategies of Chilean media on Twitter using an automatic news classifier

Matthieu Vernier ${ }^{*}$

LUIS CÁRCAMO-ULLOA ${ }^{* * *}$

Eliana ScheiHING-GarcíA ${ }^{* \star * *}$

Este trabajo da cuenta resultados iniciales del Fondecyt $\mathrm{N}^{\circ}$ 1150545 "Redes Sociales y Medios de Comunicación: Modelo de análisis basado en minería de datos para la comprensión del ecosistema informativo chileno en internet y la educomunicación ciudadana en la red". Agradecemos también la importante colaboración del Dr. Diego Sáez-Trumper del centro EURECAT, Barcelona.

** Instituto de Informática, Universidad Austral de Chile. Correo electrónico: mvernier@inf.uach.cl.

*** Instituto de Comunicación Social, Universidad Austral de Chile. Correo electrónico: Icarcamo@uach.cl.

**** Instituto de Informática, Universidad Austral de Chile. Correo electrónico: escheihi@inf.uach.cl.

\section{Resumen}

En Chile no existe una instancia independiente que publique, constantemente, estudios cuantitativos o cualitativos para comprender el ecosistema de medios tradicionales y sus adaptaciones en la Web Social. Los públicos consumen informaciones ya no sólo en periódicos y noticieros, sino también a partir de redes sociales como su fuente primaria de acceso a la información. Twitter es la red social de noticias por excelencia y los medios hacen esfuerzos por ganar adeptos en ella. En este artículo se propone una metodología basada en minería de datos web. Utilizamos técnicas de rastreo y extracción de flujos de noticias de 37 medios de comunicación chilenos que presentan una vida activa en Twitter y proponemos varios indicadores para compararlos. Analizamos los volúmenes de producción, sus audiencias potencialesy, usando técnicas de procesamiento natural del lenguaje, exploramos el contenido de la producción informativa, sus tendencias editoriales y cobertura geográfica.

Palabras clave: Medios informativos, Chile, Twitter, procesamiento natural del lenguaje, estrategias editoriales, audiencias.

\begin{abstract}
In Chile, there is no independent entity that publishes quantitative or qualitative studies that can provide the tools to understand how the traditional media environment has adapted to the social web. Nowadays, Chilean newsreaders are increasingly using social networks as their primary source of information. In this regard, Twitter plays a central role as it is considered, among the users,
\end{abstract}


as the most influential news source on social networks; consequently, mainstream media are making efforts to develop different strategies to increase their audience and influence on this platform. Nevertheless, it is possible to affirm that there is a lack of tools that can serve to analyze these strategies. The following article intends to propose a methodology based on data mining techniques to provide the tools to carry out the analysis of the new Chilean media environment. Crawling techniques were used to mine news feeds from 37 different Chilean media that are currently active on Twitter; moreover, to provide several indicators to compare them. Thus, the volumes of production were analyzed in terms of their potential audience and NLP techniques were used to explore the contents of production, their publishing standards, and their geographical coverage.

Key words: News media, Chile, Twitter, natural language processing, editorial strategies, audiences.

\section{Introducción}

En el ecosistema de medios informativos chileno, interactúan más de 2.000 concesiones de radio (Ramírez 2009), más de 100 canales de televisión con propuestas comunicacionales y cerca de 90 periódicos de prensa escrita tradicional (Corrales y Sandoval 2005). Estas cifras pueden tomarse como un punto de partida, pero si consideramos la existencia de canales locales de cable y la emergencia de medios online nos encontramos ante un escenario en expansión y poco sistematizado.

En cuanto al estado del arte en esta materia, resulta interesante consignar investigaciones como el Primer Estudio Nacional sobre Lectoría de Medios Escritos de Azocar et al. (2010) y Diarios de vida de las audiencias chilenas de Arriagada et al. (2014). El primero es un estudio académico independiente y riguroso que se reduce a los medios tradicionales escritos del país; el segundo aporta una caracterización más cualitativa de las audiencias a partir de 36 casos reportados bajo la estrategia del autoreporte.

Sabemos que en el nuevo ecosistema de medios conviven medios tradicionales, con sus señales de televisión o radio, y periódicos impresos, pero además están los medios online, entre los que cohabitan las versiones digitales de los medios tradicionales y nuevos medios nacidos para las redes con posterioridad al año 20001. Los nuevos medios, además de poner sus contenidos en formatos web, han debido adaptarse constantemente a las evoluciones tecnológicas que se proponen desde nuevas plataformas de redes sociales, nuevos dispositivos móviles y nuevos recursos de broadcasting digital.

Además de la poca sistematización de los cambios en el espacio digital chileno, existe un conocimiento muy limitado del alcance de los medios de comunicación y de la generación de audiencias. En países como España o Colombia existen organismos destinados a realizar seguimientos independientes de la realidad medial $^{2}$. En Chile, en cambio, si bien se hacen mediciones de people meter de forma permanente entre los grandes canales de televisión, dichas evaluaciones se llevan a cabo

En el año 2000 nace el periódico El mostrador, solo en formato electrónico. En el año 1994, nace La Tercera online, como espejo de su versión tradicional en papel.

Por ejemplo, http://www.aimc.es y http://www.acimcolombia.com 
a través de dispositivos distribuidos sólo en siete ciudades ${ }^{3} y$, por ende, poco pueden decir de los canales de televisión locales. En la radiofonía, las evaluaciones también son parciales, ya que, aunque la cobertura de regiones es menos centralizada y da lugar a las identidades locales, las estadísticas existentes provienen de estudios desarrollados por IPSOS ${ }^{4}$ a encargo de ARCHI (Asociación de Radiodifusores de Chile), que agrupa aproximadamente a un $50 \%$ de un total de más de 2.000 concesiones. Por su parte, los estudios de circulación y lectoría de periódicos evalúan sólo la realidad del "Gran Santiago", midiendo -en contadas ocasiones- las realidades de grandes ciudades como Valparaíso y Concepción, pero poco se sabe del impacto en el resto de las ciudades del país. Además, los estudios son encargados por la ANP (Asociación Nacional de la Prensa), que reúne a los medios más tradicionales y poderosos de Chile, y tiene por finalidad aportar información a los agentes publicitarios ${ }^{5}$.

Cabe señalar, además, que en Chile existe una particular concentración de la propiedad de los medios, que trae consigo una expresión limitada del pluralismo editorial y la escasa expresión de las realidades locales. Por otra parte, algunos estudios esporádicos de universidades u organismos independientes han dado cuenta de: a) un nivel fuerte de centralismo en las informaciones (Stambuk 1999; Puente y Grassau 2009) y b) falta de pluralismo y expresión social en los medios (Vera 2005; Vergara et al. 2012). Así, por ejemplo, el Consejo Nacional de

Ver http://www.ibopemedia.cl

Ipsos desarrolla tradicionalmente los informes de radiofonía encargados por ARCHI (Asociación de Radiodifusores de Chile). Ver http://www.ipsos.cl/ipsosradioalaire/pagcuatro.htm

5 Asociación Chilena de Agencias de Publicidad. Para mayor información, visitar http://www.achap.cl/estudios.php
Televisión (CNTV 2014) concluye que el 49\% de los encuestados entre 18 y 24 años de edad consideraron que las informaciones resultan insuficientes para entender las movilizaciones estudiantiles. Además, Maldonado, PeñaAraya y Poblete (2015) dan cuenta de que, muchas veces, los medios de comunicación mantienen también en redes sociales los sesgos de centralismo informativo tradicionales en Chile. En este contexto, donde los medios no responden a las demandas comunicativas de la sociedad, que las opiniones en redes sociales emergen con potencia y pueden llegar a generar comunidades informativas alternativas y, en alguna medida, se desarrollan procesos comunicativos sobre las opiniones de multitudes, en un sentido similar a lo que Baeza-Yates y Sáez-Trumper (2015) definen como sabiduría de multitudes en redes sociales.

\section{Justificación y estado del arte}

\section{1. Cambios en el contexto digital}

En un contexto en el que, por una parte, la sociedad chilena presenta una fuerte adherencia al uso de redes sociales y, por otra, los medios de comunicación configuran estrategias específicas de community manager para redes sociales, resulta interesante desarrollar una herramienta que permita escanear el ecosistema informativo chileno, categorizar la información circulante y visualizar características del flujo informativo chileno. Por ello, desarrollamos una serie de herramientas que permiten observar el flujo de informaciones de 37 medios informativos ${ }^{6}$ desde la red social Twitter. Entre los medios

Esta cifra de medios responde a la etapa inicial del proyecto, pero en la actualidad ya se pueden seguir más de 250 medios de comunicación chilenos. 
seguidos se encuentran "medios tradicionales" -como El Mercurio On Line, La Tercera, Mega, TVN, entre otros que nacieron previos a Internet y se adaptaron a la misma- y "nuevos medios" -tales como El Mostrador, El Desconcierto y Pulso- que nacieron exclusivamente en Internet.

Imaginemos que la forma primera de informarse son las redes sociales. Cada mañana los ciudadanos revisan la prensa desde Twitter o Facebook y valoran, comentan y comparten informaciones hacia sus círculos sociales. Desde ahí, y a lo largo del día, se generan lecturas y opiniones que se encuentran mediadas ya no sólo por los medios de prensa que aportaron las informaciones, sino también por las interacciones viralizadoras que familiares, amigos o conocidos ejecutan al valorar, reponer y comentar noticias en las redes sociales. Con todo, Internet ha cambiado la forma de informarse de los ciudadanos, y el poder de la audiencias y los grandes medios se están reconfigurando con nuevas reglas (SáezTrumper 2011).

Si bien no se puede asumir que las redes sociales sean el mecanismo mayoritario utilizado para informarse por toda la población, sí existen antecedentes concretos (Newman 2013) de que en el mundo se construye una tendencia en la que los sujetos menores de 35 años consideran que Internet es la mejor forma de acceder a noticias, mientras que el grupo sobre esa barrera etaria (mayores de 35 años de edad) tiende a preferir informarse más tradicionalmente vía televisión. Además, se puede inferir que, probablemente, con el paso de las generaciones, la frontera de 35 años se desplazará.

Chile demuestra ser un país que adopta con facilidad las tendencias derivadas de las Tecnologías de la Información y las Comunicaciones, y tanto ComScore (2013) como Interactive Advertising Bureau O IAB (2012) señalan que la penetración del social media alcanza aproximadamente al $80 \%$ de la población. Estudios recientes de Cárcamo-Ulloa y Sáez-Trumper (2014) dan cuenta de que las interacciones con medios de prensa en Facebook pueden tener un volumen similar en España y en Chile, con la salvedad de que la población de nuestro país representa aproximadamente un $35 \%$ de la de España. Además, Halpern (2014) detectó en Chile una interesante tendencia al consumo de informaciones interactuando entre pantallas (por ejemplo, ver TV y utilizar RRSS al mismo tiempo).

Los nuevos ecosistemas de la información (Díaz-Nosty 2013) incluyen como agentes relevantes de comunicación a las redes sociales. Cardoso (2014) señala que los "medios sociales" han cambiado el modelo de comunicación, "no solo porque técnicamente han multiplicado las formas en que podemos apropiarnos de la comunicación en cuestión o elegir relacionarnos de forma determinada, sino también porque son el eslabón perdido necesario para operar una revolución en nuestro modelo de comunicación" (Cardoso 2014: 18).

Por su parte, los medios de prensa tradicionales también incorporan herramientas como Facebook y Twitter a modo de soportes divulgativos de las informaciones que cuelgan en los website (Tejedor-Calvo 2010), con el fin de viralizar sus contenidos informativos y de entretenimiento. Además, en el día a día, las rutinas de los medios de prensa hacia sus redes sociales resultan más o menos reproductivas de sus informaciones y aparece en las redacciones la figura del Community Manager 
o el Social Media Editor (Sánchez y Méndez 2013). Sin embargo, gran parte de la actividad viral de las informaciones depende también de las interacciones que los propios lectores/ usuarios ejecutan valorando (like), comentando (comment) y compartiendo (share) contenidos desde y hacia redes sociales como Facebook (Cabalin 2014), o utilizando el retweet en Twitter.

Esta última situación trae consigo un cambio importante a nivel de audiencias, pues, por un lado, los liderazgos informativos en redes sociales pueden no reproducir exactamente los ranking de lectorías y/o audiencias tradicionales (Cárcamo-Ulloa y Sáez-Trumper 2013), mientras que, por otro lado, permitirían la irrupción de nuevos medios alternativos. Además, un medio en Internet deja de ser sólo una radio, un periódico o una televisora y se convierte en un difusor de distintos contenidos informativos multimodales (Pardo 2012a, 2012b) o hipertextuales, interactivos y multimediales (Masip et al. 2010). Una prueba de ello es que hoy el contenido más viralizado de una radio emisora online puede ser un video recuperado de YouTube o aportado por un reportero ciudadano.

\section{2. ¿Por qué la minería de datos web?}

La herramienta para describir un mapa real de los medios en el nuevo ecosistema informativo de las redes sociales existe, y tiene más de tres décadas de desarrollo de la mano de los investigadores en ciencias de la computación: se trata de la minería de datos web. Dichos estudios se han instalado en áreas como la bibliotecología (Peters 1993; Kurth 1993; García, Botella y Marcos 2010; Marcos y González 2010) y, además, en los entornos virtuales adaptativos (Morales, Soto y Martínez
2005) existe una valoración creciente del Web Mining como aporte a la búsqueda de patrones para el mejoramiento de los procesos comunicacionales involucrados. En tal sentido, Baeza-Yates (2009) y Baeza-Yates y Passi (2011) ofrecen una panorámica que revisa las principales tendencias de expansión para la minería de datos en la Web 2.0, el spam, análisis de búsquedas, redes sociales y la privacidad en internet.

En el ámbito de la comunicación social existen experiencias relevantes en el análisis apoyado en la minería de datos en Europa y Estados Unidos (Yang y Leskovec 2011), las que aportan una perspectiva internacional (Sáez-Trumper, Castillo y Lalmas 2013). Sin embargo, en Chile hacen falta diagnósticos locales que den cuenta de la expansión de los medios de comunicación en los últimos años $\mathrm{y}$, además, permitan conocer sus niveles de influencia en las redes sociales. Así por ejemplo, en el catastro realizado por mediaonline.net se describen 296 medios para el caso chileno. Ellos se distribuyen irregularmente en la geografía, con una lógica de mayor concentración en la Región Metropolitana y sus regiones vecinas, y menor despliegue hacia los extremos norte y sur del país. Sin embargo, se conoce muy poco sobre los impactos de la producción de información de los medios alternativos y/o regionales y sus potenciales audiencias.

Pese a que, evidentemente, Chile no presenta una realidad de cultura digital homogénea y que existen comunidades que aún se encuentran aisladas de la sociedad de la información, es claro que la brecha digital está hoy en los usos más que en los accesos. Es por eso que el presente trabajo tiene por objetivo proponer un modelo de análisis basado en minería de datos en 
redes sociales de medios de prensa en la Internet chilena. Esta propuesta entremezcla estrategias metodológicas basadas en las minería de datos web (Baeza-Yates 2009), específicamente el seguimiento de las interacciones en los social media (Cárcamo-Ulloa y Saez-Trumper 2013), hacer minería de textos para clasificar estas noticias (Sáez-Trumper, Castillo y Lalmas 2013; Vernier, Monceaux y Dille 2012) y la detección de temas emergentes en Twitter (Guzmán y Poblete 2013).

\section{Metodología del estudio}

\subsection{Datos y procedimientos de análisis}

Se realizó un procedimiento que permitió seguir todas las informaciones emitidas en Twitter por 37 medios de comunicación chilenos ${ }^{7}$. El crawler inicial almacena las informaciones en una base de datos no SQL y, como cada tweet contiene un hipervínculo a la información extendida en una página web, también se realiza un segundo proceso de crawling to link a la noticia para aplicar una limpieza o scraping de contenido relevante de la web. Para construir la infraestructura de minería y análisis de datos se recurrió a herramientas tales como Elasticsearch, Kibana y UIMA.

Los datos se obtuvieron entre el 01 de junio de 2015 y el 30 de noviembre de 2015 para contemplar un semestre móvil. Los medios emitieron 736.538 noticias vía sus cuentas de Twitter: junio, 120.571; julio, 119.704; agosto, 124.910; septiembre, 103.739; octubre, 123.952 y noviembre, 129.042 .

Como procedimientos de análisis para este artículo realizamos: a) un primer paso de estadística descriptiva de la producción de noticias y de la cantidad de seguidores que tiene cada una de las cuentas de Twitter sobre 6 meses de emisiones informativas, y b) un procedimiento de clasificación de noticias

Figura 1. Representación del modelo de obtención de datos

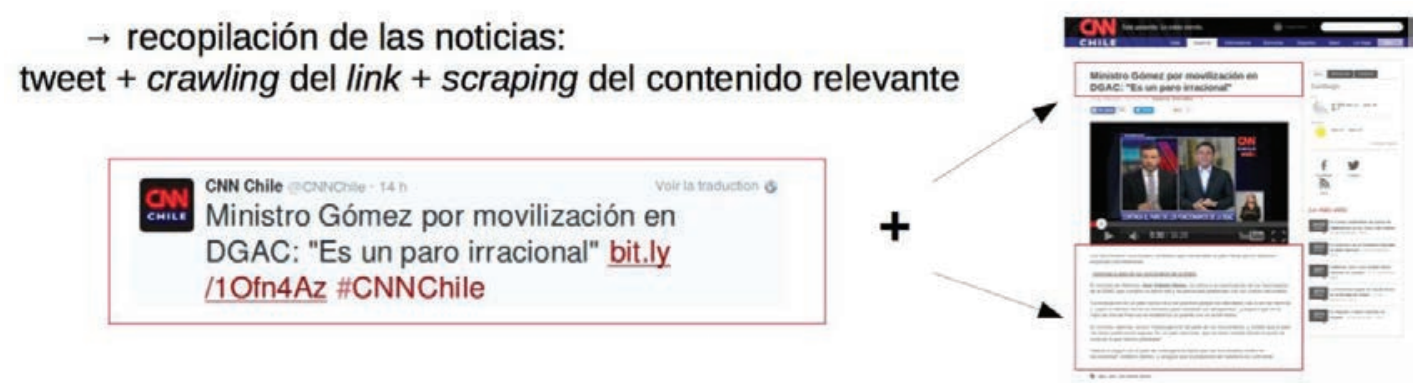

Fuente: elaboración propia.

Para obtener mayores detalles metodológicos de la investigación, revisar o contactar a los investigadores a través del sitio web www.migracionescomunicativas.cl 
mediado por minería de texto y tratamiento automático del lenguaje sobre la producción informativa del mes de octubre de 2015. Se decidió hacer un experimento acotado a un mes para probar experimentalmente algoritmos que permitan luego procesar mayores volúmenes de información.

En la primera etapa, los procesos de crawling y scraping permitieron la extracción de metadatos (fechas y nombres de medios, principalmente) de 736.538 informaciones compiladas. En la segunda etapa, apoyados en la herramienta UIMA (Unstructured Information Management Architecture), se realizaron pretratamientos informatizados propios de la programación de lenguaje natural: a) tokenización, b) análisis gramatical, c) lematización, y d) extracción de palabras clave. En primer lugar, la tokenización o proceso de análisis lexicográfico permite discriminar el conjunto de posibles secuencias de caracteres que constituyen un token o lexema. En segundo lugar, el análisis gramatical automatizado permite identificar la categoría gramatical de las palabras (nombres, adjetivos, verbos, etc.), por ejemplo: "Vamos a seguir con el plan de contingencia", donde "vamos" es el verbo y no debe confundirse con Vamos, la ciudad en Grecia. Para este caso, el algoritmo de clasificación de secuencias escogido es Hidden Markov Model (HMM), y para el caso del español el modelo se ha entrenado con el corpus "Spanish CRATER". En tercer lugar, la lematización (lema se refiere al ítem lexical) permite generalizar la forma de las palabras, por ejemplo: movilizaciones se puede generalizar como movilización; acusó se generaliza como acusar, etc. En este caso se utiliza un algoritmo basado en reglas específicas para el español. En cuarto lugar, la extracción de palabras clave ayuda a identificar las palabras que mejor resumen el texto, utilizando: a) una lista de frecuencias de palabras del español de Chile (Sadowsky 2012), y b) un algoritmo basado en la medida TF-IDF, que relaciona la frecuencia de aparición de un tema en un texto determinado respecto de su frecuencia en una colección de documentos.

Una vez realizado el pre-procesamiento de los datos, se realiza un análisis de las noticias a partir de dos nuevos procesos: a) la clasificación del tema de las noticias mediante un algoritmo de aprendizaje supervisado, y b) la extracción y desambiguación de los nombres de localidades mencionados en la noticia. El proceso de análisis y visualización completo se describe en la Figura 2.

\subsection{Metodología para clasificar el tema}

El corpus de entrenamiento utilizado es Wikinews ES, que consiste en 500 noticias etiquetadas en español. Además, se considera la representación vectorial de los textos: lista de lemas y su TF-IDF. El algoritmo de aprendizaje supervisado utilizado para la clasificación es Support Vector Machine (SVM) (Joachims 2002) con la herramienta Weka (Waikato Environment for Knowledge Analysis).

Evaluación del clasificador: El clasificador se evalúa sobre el corpus de entrenamiento (validación cruzada considerando 10 conjuntos), calculando las medidas de precisión y exhaustividad.

Precisión (C): Es la tasa de textos clasificados en la clase $\mathrm{C}$ por el clasificador (bien clasificados), versus textos clasificados $\mathrm{C}$ por el clasificador. Esta medida permite cuantificar el ruido en la clasificación. 
Figura 2. Esquema de extracción, análisis y visualización de datos textuales

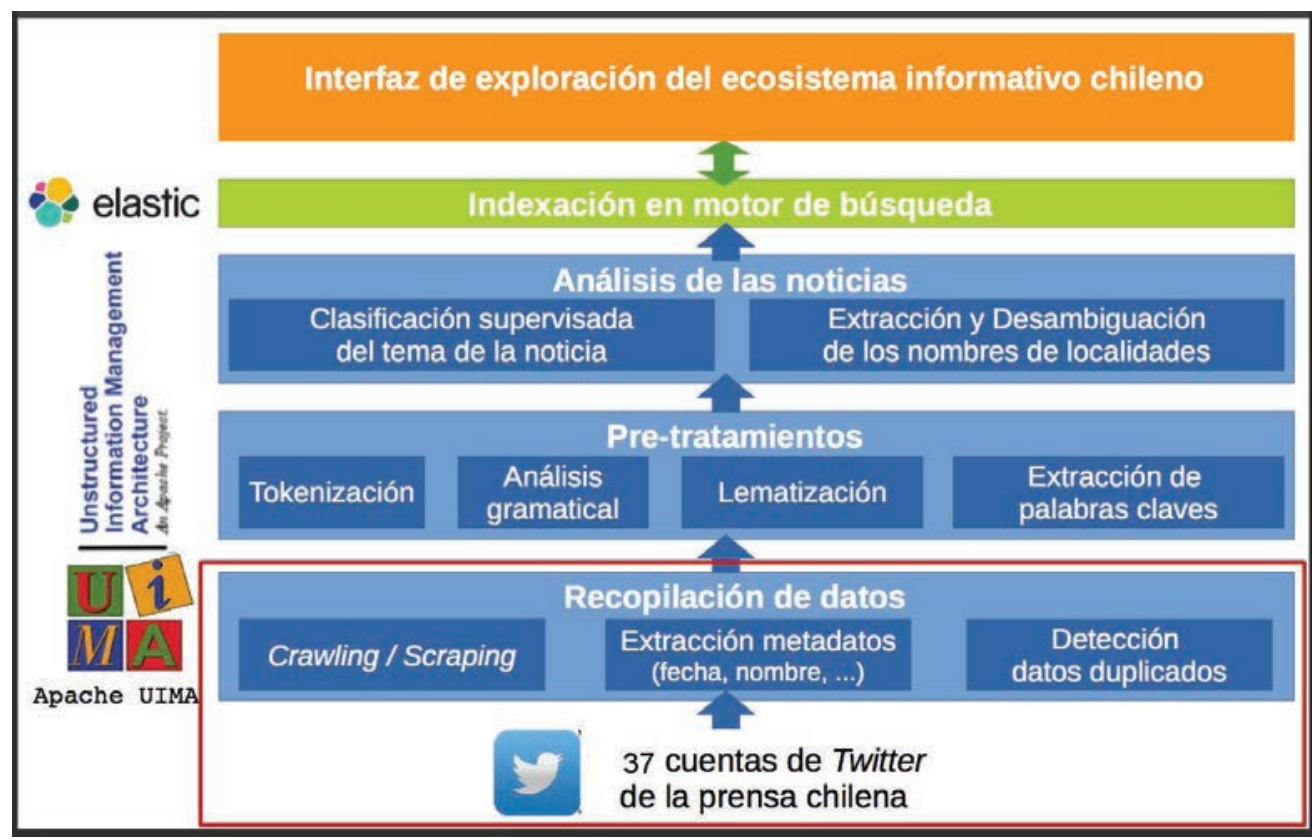

Exhaustividad (C): Es la tasa de textos clasificados en la clase $\mathrm{C}$ por el clasificador (bien clasificados), versus textos clasificados $\mathrm{C}$ en realidad. Esta medida permite cuantificar el silencio en la clasificación.

\subsection{La extracción de localidades}

Además de la necesidad de clasificar los temas de referencia de la prensa chilena, resulta interesante conocer si cuando se alude a esos temas se hace referencia a hechos noticiosos ocurridos en una variedad de lugares geográficos o si existe una concentración homogénea de referentes. Para el proceso de extracción de los nombres de las localidades se aplicó un segundo nivel de análisis del contenido textual, bajo las siguientes reglas metodológicas:
Contraste frente a recurso GeoNames.org: Se trata de una base de datos geográfica multilingüe, entre cuyas entradas se encuentran: países, regiones, ciudades, pueblos, lagos, calles, etc., y a su vez presenta coordenadas geográficas de latitud y longitud.

Indexación de los nombres de localidades en español (MongoDB - 7 Go): Que contiene países (197), ciudades o pueblos ( 3.000.000), contrastando en base a un algoritmo de búsqueda de palabras/grupos de palabras en las noticias.

\subsection{Desambiguación}

Evidentemente, la identificación de localidades no está exenta de ambigüedades como, por ejemplo: el mismo nombre de la ciudad chilena 
Valdivia puede tener lugar en otros países del mundo (Colombia, Uruguay, Ecuador, etc.). A su vez, muchas palabras en español corresponden a ciudades existentes en el mundo, por ejemplo: la conjugación de tercera persona plural del verbo caer "caen", es un lexema idéntico a la ciudad francesa Caen. Ante estas ambigüedades conservamos solamente ciudades/pueblos que se encuentran en el país de la noticia.

Finalmente, gracias a Elasticsearch y Kibana, se pudo realizar la indexación de las informaciones compiladas y procesadas. Con ello fue posible ofrecer una interfaz de exploración del ecosistema informativo de medios chilenos en Twitter, lo que facilita la visualización de datos con distintas características.

\section{Resultados}

A continuación presentamos los diferentes resultados del análisis realizado. Abordaremos la producción de información en el tiempo, las tendencias temáticas y la cobertura geográfica de los hechos noticiosos.

\subsection{Volúmenes, ciclos de producción de información y audiencias potenciales}

Los datos capturados dan cuenta de una emisión mensual promedio de 120.259,6 tweets. La mayor variación corresponde a septiembre, pues se emitieron 103.739. Dicha baja se explica, básicamente, porque septiembre presenta una mayor cantidad

Figura 3. Interfaz de visualización de datos

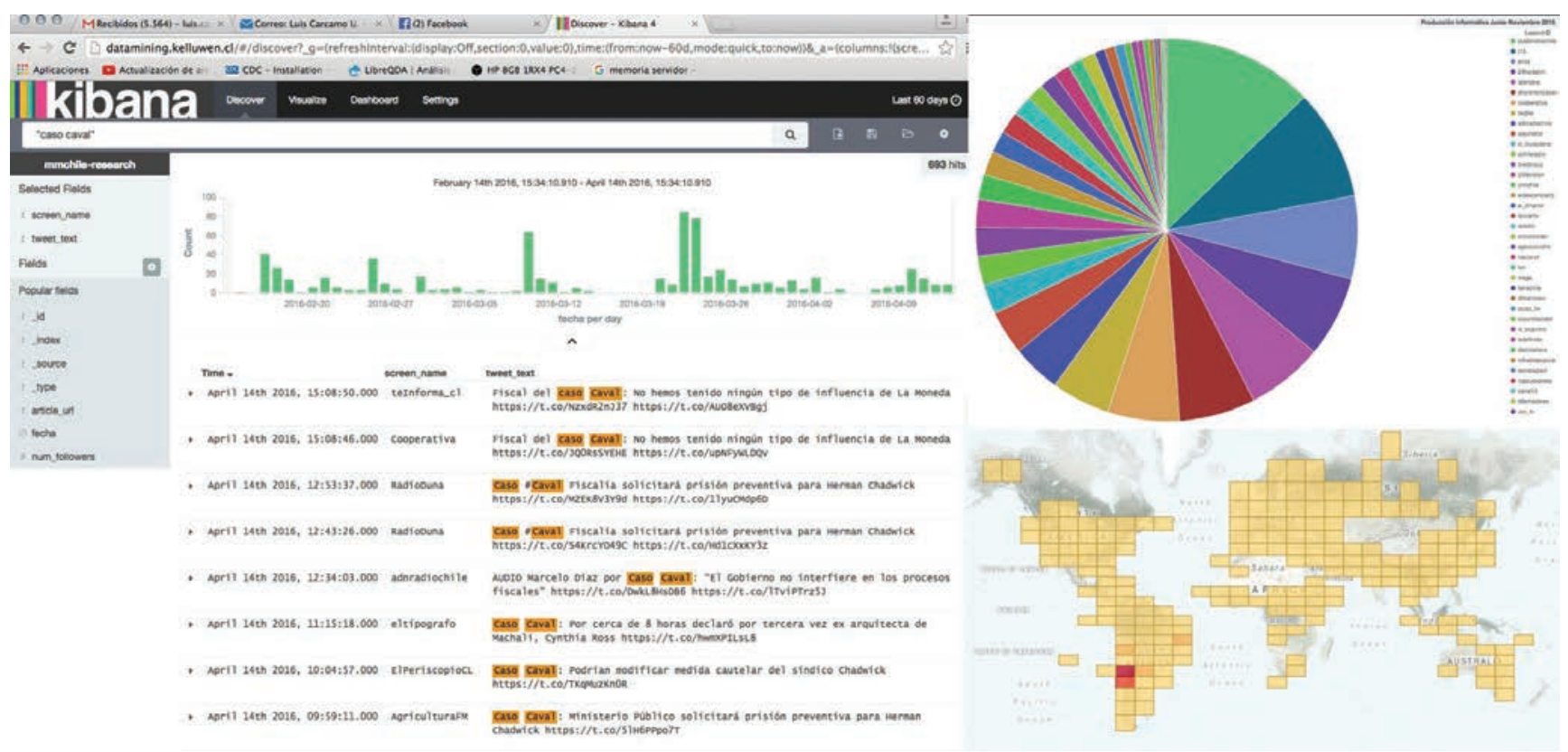


de días no laborables, correspondientes a los feriados de fiestas patrias y, con ello, las dinámicas de los turnos de prensa parecen disminuir entre los responsables de los social media. Efectivamente, los datos compilados en los días feriados para algunos medios fueron igual o cercano a 0 . Al margen de la incidencia de septiembre, en el Gráfico 1 se aprecia cierta estabilidad en la emisión de tweets por parte de los medios de comunicación.

Haciendo un zoom en los datos, se eligió analizar octubre de 2015, observándose que los 37 medios seguidos por el crawler produjeron 123.952 emisiones en Twitter. De ese total, 65.572 correspondían a noticias diferentes para ese mes, con un promedio aproximado de 2.000 por día. La diferencia se explica por una tendencia de algunos medios a repetir emisiones con una misma noticia, tweets sin un link o con un link roto que no se pudo minar. La producción de informaciones tuvo un comportamiento bastante estable con incrementos de lunes a viernes y descensos en los fines de semana. Se marcan claramente dos excepciones dadas por los enfrentamientos de la selección chilena de fútbol en las eliminatorias del mundial, eventos que se reflejan los días 8 y 13 de octubre de 2015 (Gráfico 2).

El Gráfico 3 expone que los medios con mayor cantidad de emisiones son medios que tienen

Gráfico 1. Emisión global de tweets entre junio y noviembre de 2015

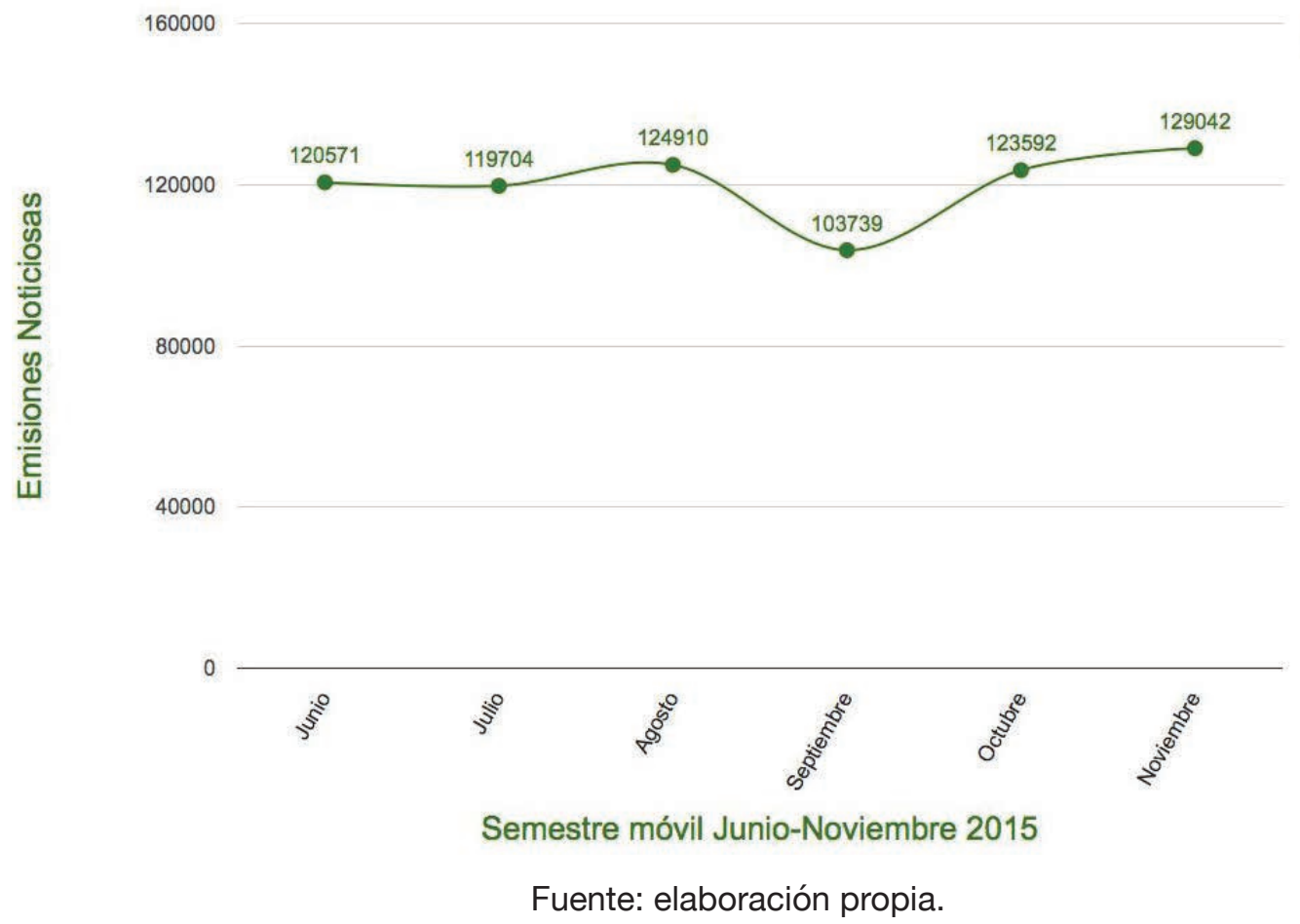


Gráfico 2. Emisión de informaciones en octubre de 2015

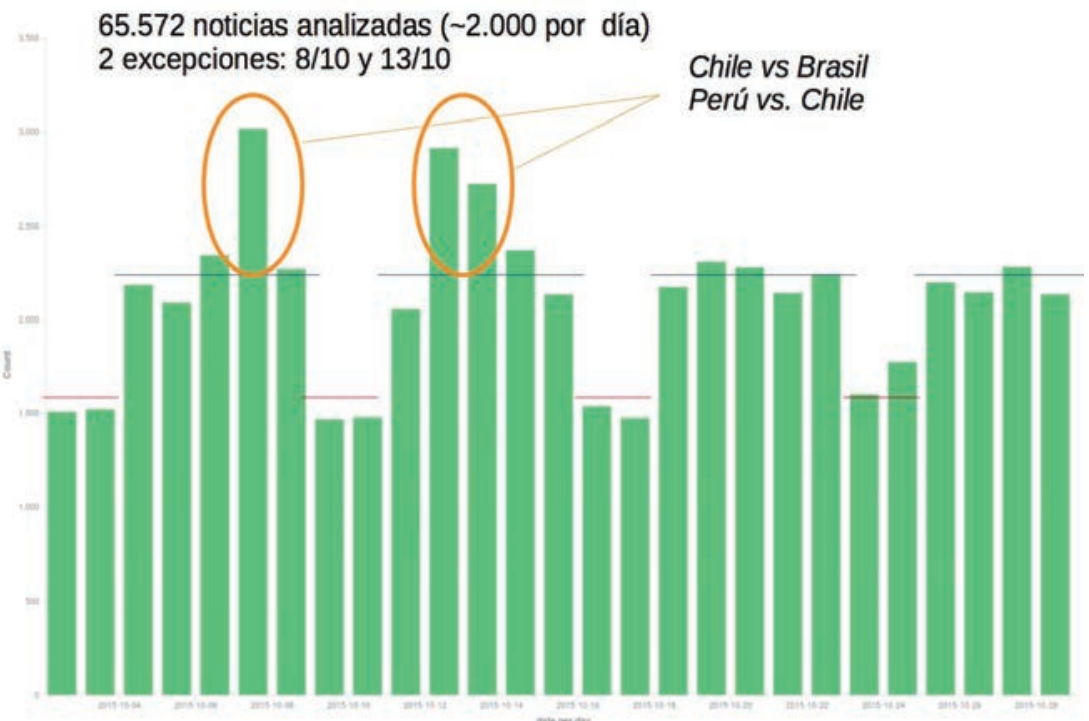

Fuente: elaboración propia.

Gráfico 3. Emisión de tweets por cada medio entre junio y noviembre de 2015

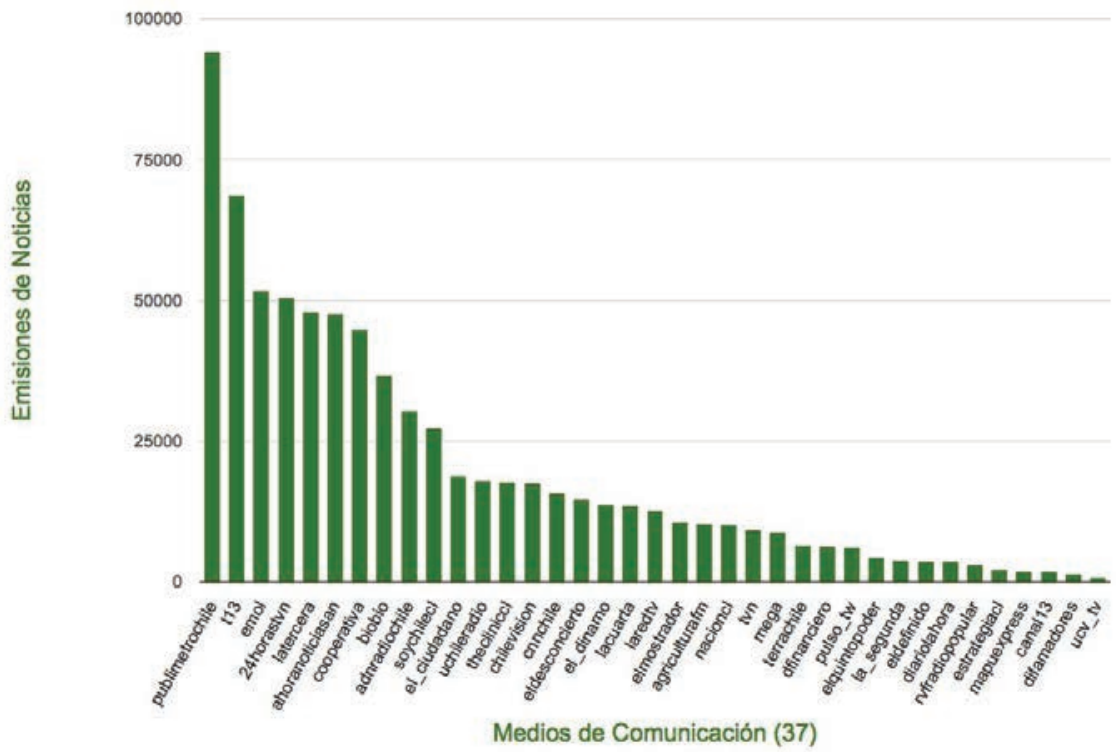

Fuente: elaboración propia. 
producción análoga y digital, aunque se puede observar una variabilidad en la que alternan medios de prensa en papel y programas televisivos (Tele 13, Canal 24 Horas y Ahora Noticias), radioemisoras (Cooperativa, Bio Bio y ADN Radio Chile) y prensa de papel (Publimetro, Emol, La Tercera y Soy Chile). Se puede apreciar cómo se concentra la emisión de informaciones hacia Twitter en los 10 primeros medios. Medios alternativos tales como El Ciudadano, Radio Universidad de Chile, The Clinic y El Desconcierto, El Dínamo y El Mostrador ocupan un lugar secundario en términos de producción de información.

Otra variable importante de revisar dice relación con las potenciales audiencias de los medios de comunicación, que no necesariamente se correlaciona con la producción de información que los medios hacen. El Gráfico 4 permite observar que, de acuerdo al número de seguidores, se reconocen claramente tres grupos de medios. La primera clase de medios supera los 2.000.000 de seguidores y en ella participan medios como Canal 24 Horas, TVN, Tele13, CNN Chile, Bio Bio y Cooperativa. En una segunda clase cohabitan los medios que tienen más de 500.000 seguidores, y en ella están La Tercera, Emol, ADN Radio Chile, Chilevisión, La Cuarta, Ahora Noticias, El Mostrador, The Clinic, Terra, Publimetro y Mega. En una tercera clase, con menos de 500.000 seguidores, participan La Segunda, La Red, La Nación, La Hora, El Dínamo, El Ciudadano y Soy Chile, entre otros.

Gráfico 4. Clases de medios según número de followers en Twitter

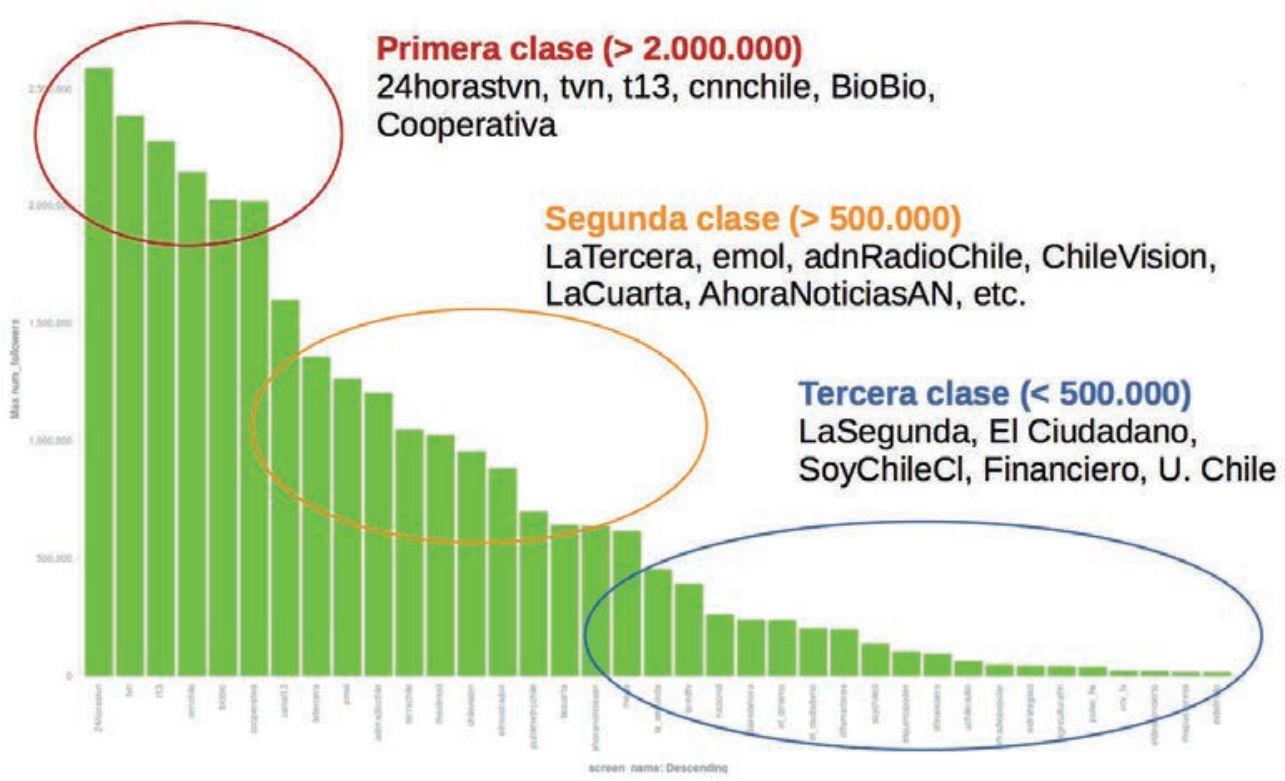

Fuente: elaboración propia. 


\subsection{Análisis mediante clasificación temática de las noticias}

En una primera fase, se utilizaron los datos de entrenamiento descritos en la metodología, que permitieron probar un modelo de clasificación de las noticias en los siguientes diez temas posibles: accidentes, deportes, ecología, economía, entretenimiento, judicial, política, salud, sociedad, tecnología. Como resultado de este entrenamiento, se obtuvo un modelo de clasificación cuya validez se puede apreciar en la Tabla 1, presentando las medidas de precisión y exhaustividad calculadas. Se puede observar que el modelo se comporta con mayor precisión en los temas de salud, judicial y accidentes, y con mayor exhaustividad en los temas de deporte y salud.

Con este modelo se procedió a la clasificación de noticias del mes de octubre de 2015 (65.572 noticias minadas). El proceso permitió observar que más del $50 \%$ de las noticias difundidas por los medios de comunicación en Twitter corresponden a Deporte + Entretenimiento (Gráfico 5). Se trata de una situación que se repite al interior de la mayoría de los medios de comunicación seguidos, exceptuándose de esta tendencia medios como: El Desconcierto, Radio Universidad de Chile, El Mostrador, El Financiero, Agricultura FM, Pulso y Diario Estrategia.

Si bien la tendencia editorial mayoritaria está marcada por el gran número de medios que dedican más del $50 \%$ de su producción a deporte y entretenimiento, también se puede observar una segunda tendencia que releva sobre un $25 \%$ de su producción a Economía + Política (Gráfico 6). En esta línea se ubican medios tales como La Tercera, Radio Bio Bio, El Desconcierto, Radio Universidad de Chile,

Tabla 1. Validación de precisión y exhaustividad de la clasificación de noticias

\begin{tabular}{|c|c|c|}
\hline Temas & Precisión & Exhaustividad \\
\hline Accidentes & $95,8 \%$ & $88,5 \%$ \\
\hline Deportes & $89,0 \%$ & $98,1 \%$ \\
\hline Ecología & $71,4 \%^{*}$ & $83,3 \%$ \\
\hline Economía & $64,4 \%^{*}$ & $76,3 \%^{*}$ \\
\hline Entretenimiento & $77,8 \%^{*}$ & $84,0 \%$ \\
\hline Judicial & $97,0 \%$ & $88,9 \%$ \\
\hline Política & $91,7 \%$ & $84,6 \%$ \\
\hline Salud & $100 \%$ & $96,3 \%$ \\
\hline Sociedad & $88,2 \%$ & $83,3 \%$ \\
\hline Tecnología & $94,7 \%$ & $75,0 \%{ }^{*}$ \\
\hline
\end{tabular}

*Valores cercanos al $75 \%$

Fuente: elaboración propia. 
Gráfico 5. Distribución de las informaciones en octubre de 2015. Tendencia Deporte + Entretenimiento

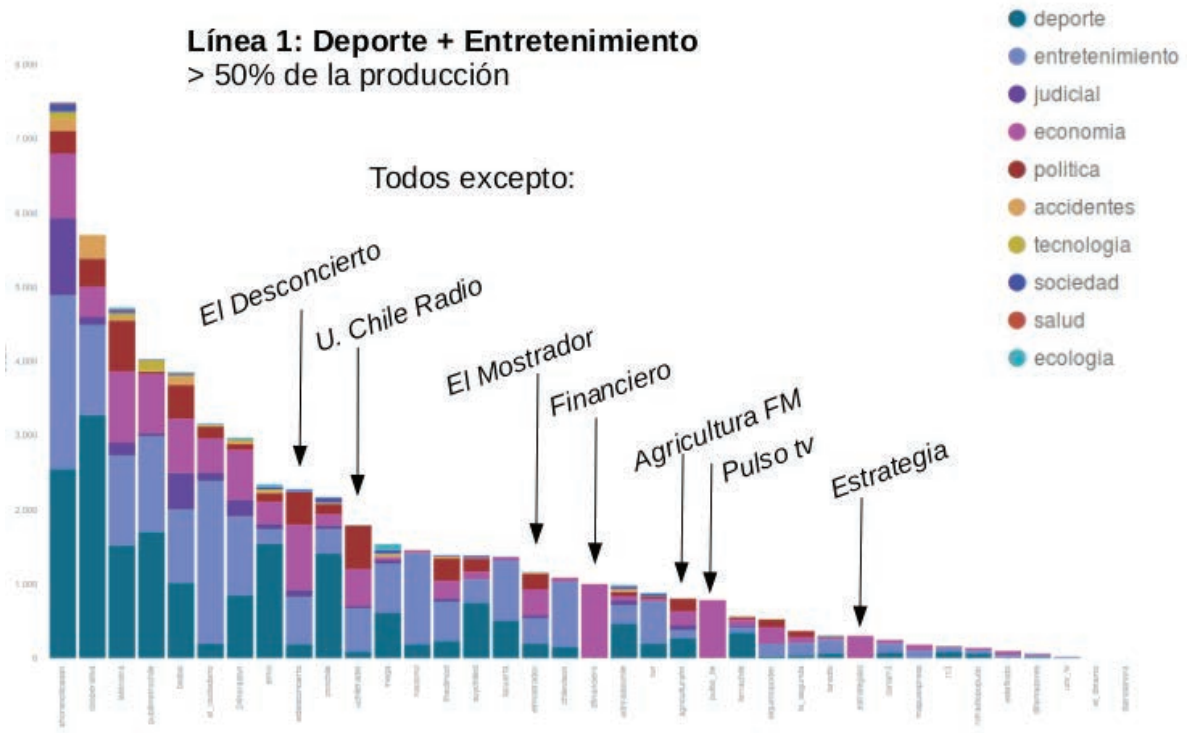

Fuente: elaboración propia.

Gráfico 6. Distribución de las informaciones en octubre de 2015. Tendencia Economía + Política

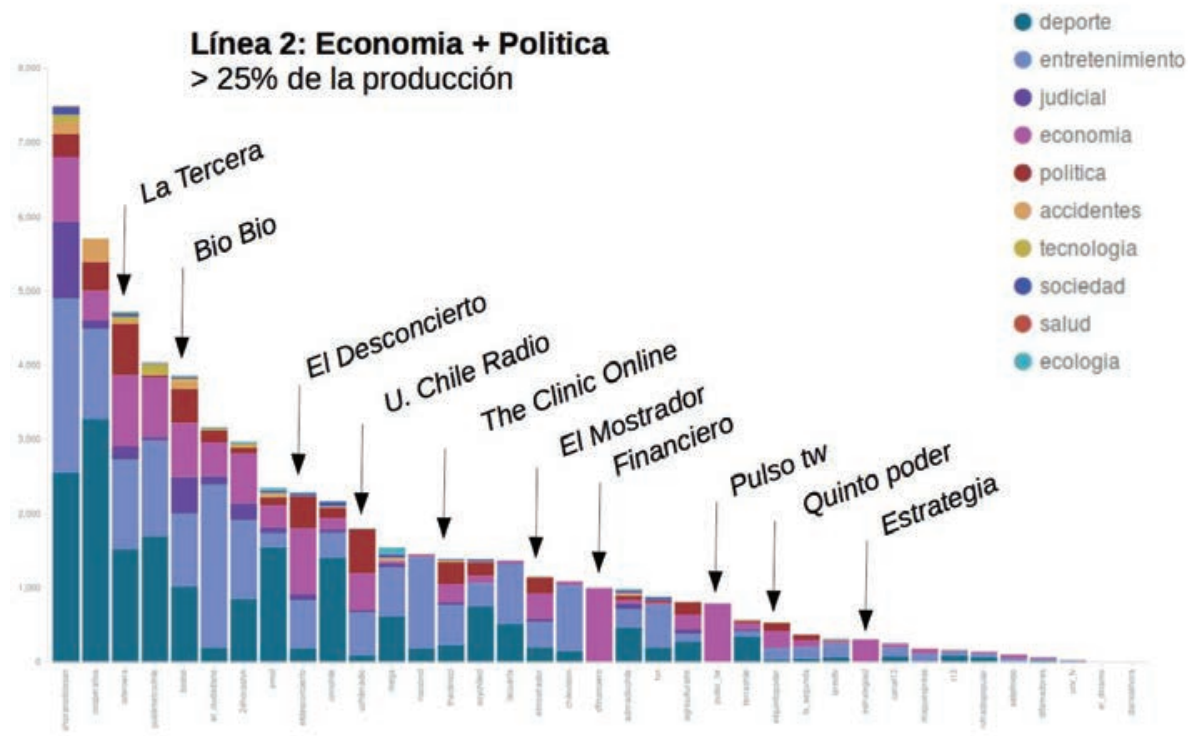

Fuente: elaboración propia. 
The Clinic, El Mostrador, El Financiero, Pulso, El Quinto Poder y Diario Estrategia.

Se presenta también una tercera tendencia de medios que dedican más de un $10 \%$ de su producción informativa a eventos judiciales (homicidios, tráfico de drogas, asaltos, entre otros). En este grupo se ubican Ahora Noticias, Radio Bio Bio y ADN Radio Chile (Gráfico 7).

\subsection{Cobertura geográfica de la información}

Los datos del mes de octubre de 2015 dieron cuenta de una esperable centralización de las noticias que refieren a Santiago de Chile, pero también se expresa cómo un fenómeno natural (temblor en La Serena) es capaz de llamar la atención de las pautas informativas, o cómo eventos de deportes y entretenimiento son capaces de llamar fuertemente la atención de los medios chilenos aun cuando sucedan en otro país, por ejemplo, Argentina (Mapa 1). De la misma forma, queda en evidencia la ausencia de informaciones que refieran a las regiones de Aysén y Magallanes.

A nivel global se pudo observar que los medios chilenos incluyen en sus pautas informativas noticias de gran parte del mundo (Mapa 2). Se constata, como era esperable, un fuerte componente de noticias nacionales con énfasis en el centro del país. La presencia de noticias

Gráfico 7. Distribución de las informaciones en octubre de 2015. Tendencia Judicial

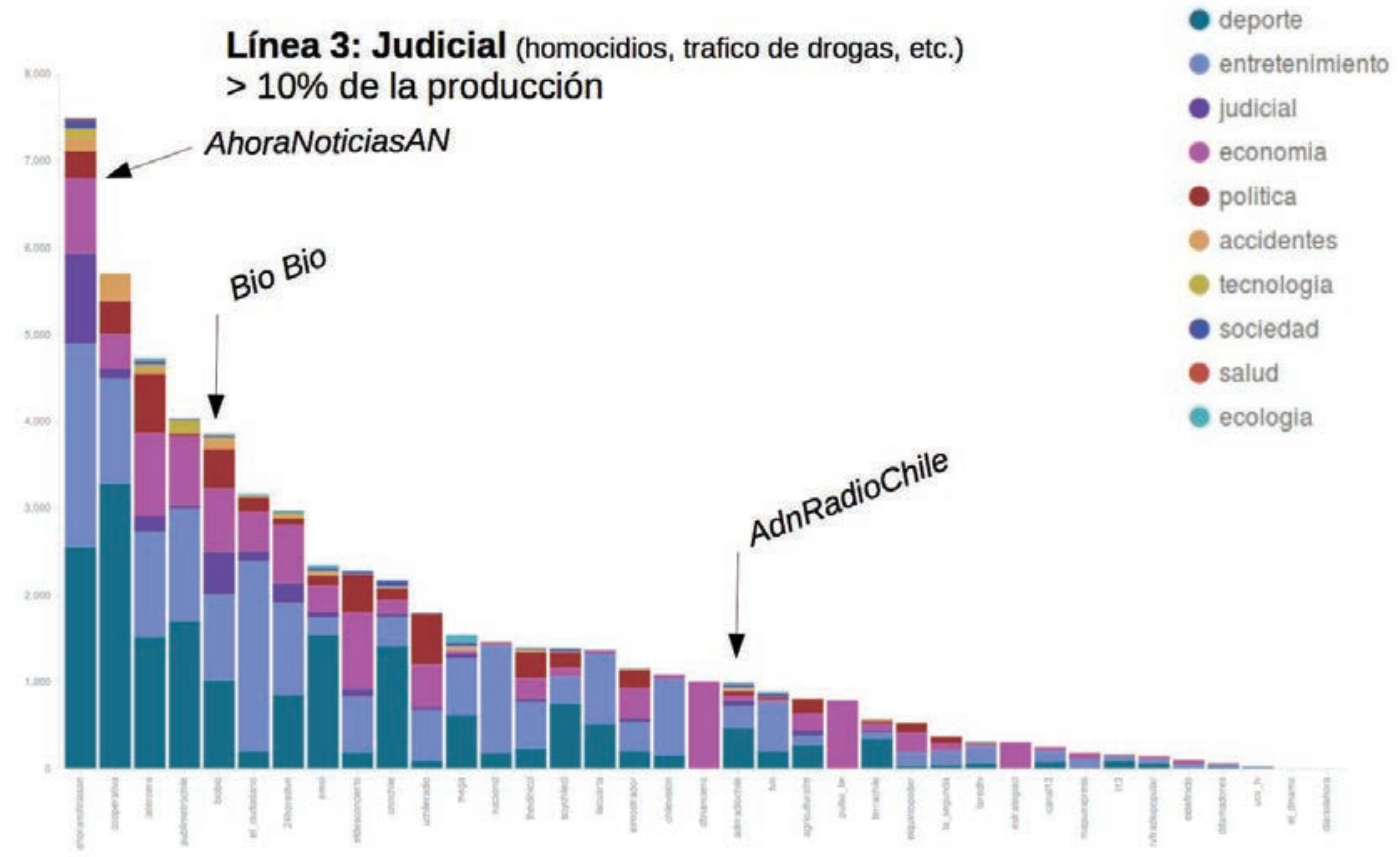

Fuente: elaboración propia. 
Mapa 1. Distribución geográfica de las informaciones en octubre de 2015

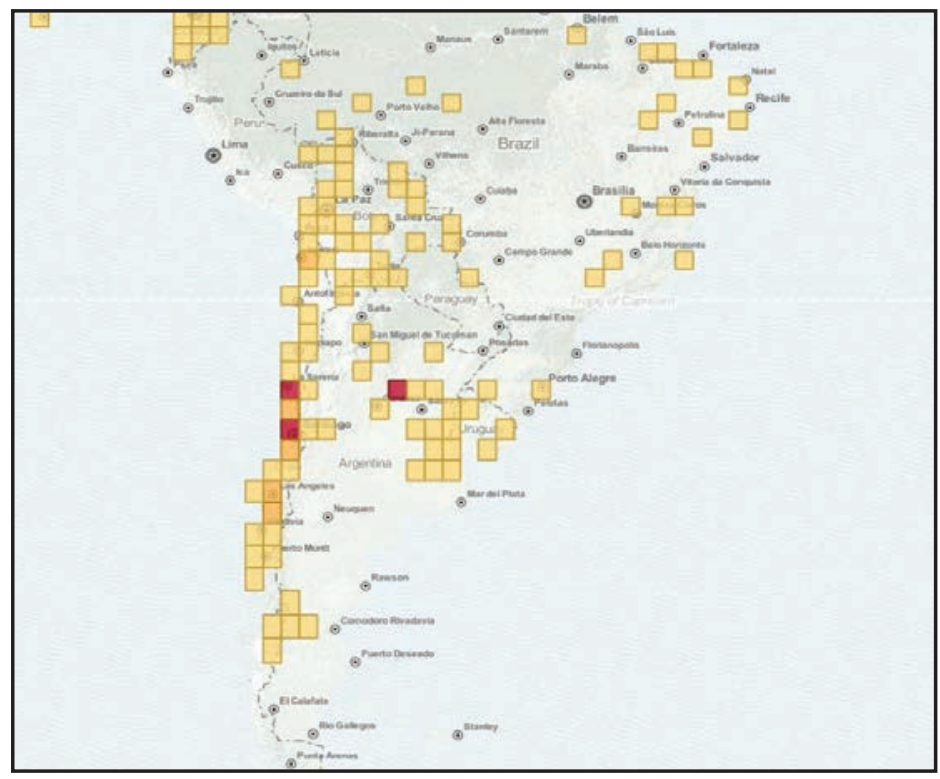

Fuente: elaboración propia.

Mapa 2. Distribución geográfica de las informaciones en octubre de 2015

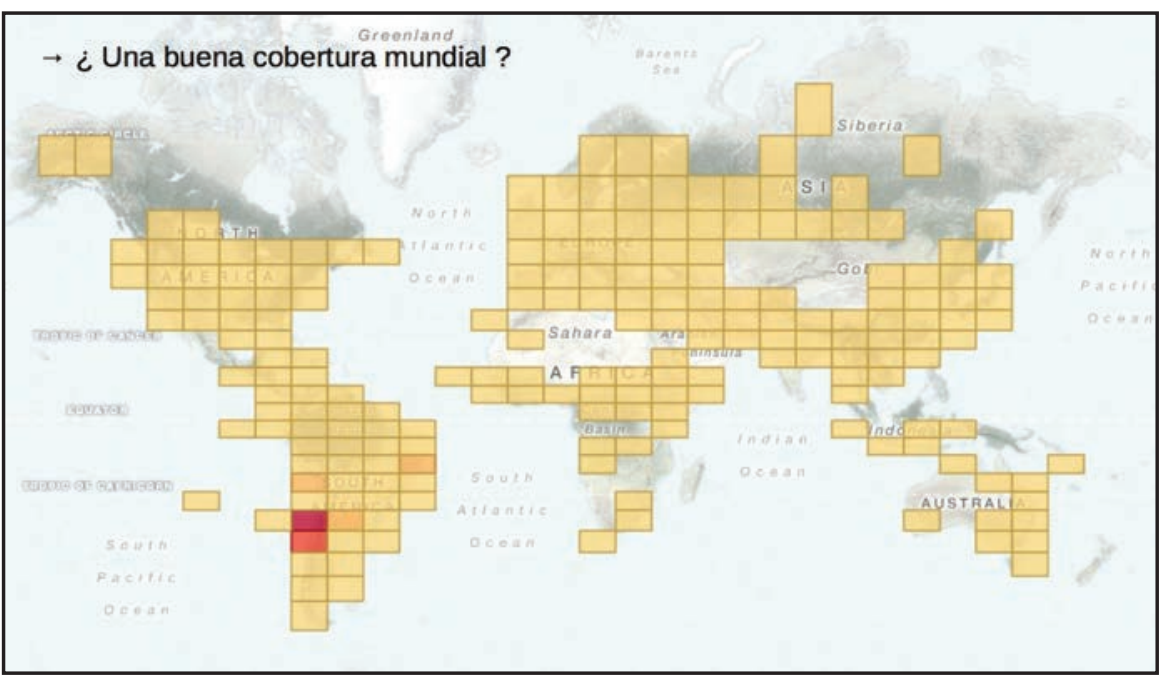

Fuente: elaboración propia. 
referentes a Perú y Brasil se explica por las eliminatorias al mundial (fenómeno también reflejado en el Gráfico 2). Finalmente, se expresa una considerable cantidad de noticias referidas a Argentina (tendencia que se explica por una conjunción de noticias deportivas, políticas y de farándula de dicho país que involucraban también a personajes chilenos).

Evidentemente, la configuración de la cobertura global se constituye de las coberturas parciales de los distintos medios chilenos. Así, por ejemplo, sobre temas de política, La Tercera ostenta una cobertura geográfica mayor que Radio Cooperativa, tal como se observa en los Mapas 3 y 4 . Ambos mapas dan cuenta de las noticias que el clasificador identificó en la categoría "política" y para la que cada medio publica noticias referentes a distintos lugares del globo.

\section{Conclusiones}

Siendo un primer estudio en el contexto de una investigación con un plazo de cuatro años (2015-2016), ya es posible hacer una serie de observaciones generales:

- Existe una gran disparidad en la emisión de informaciones que hacen los medios de comunicación del ecosistema informativo chileno: 10 medios producen el $68 \%$ de las emisiones y 27 generan el $32 \%$ de los tweets en el período junio-noviembre de 2015.

- La producción de la información no se correlaciona directamente con las potenciales audiencias alcanzadas. Por ejemplo, un medio como Publimetro, que emite muchos contenidos diariamente, no se instala como uno de los medios con mayor cantidad de seguidores en Twitter.

Mapas 3 y 4. Distribución geográfica de las informaciones según las localidades del mundo mencionadas por Cooperativa y La Tercera

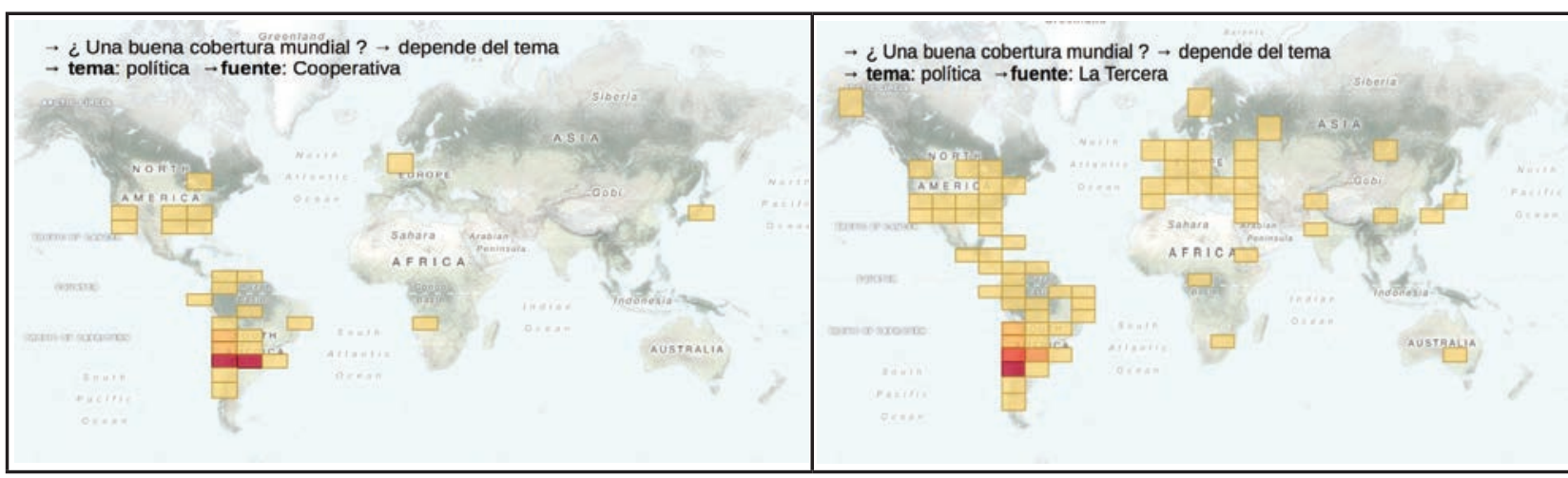

Fuente: elaboración propia. 
- Hasta ahora podemos distinguir tres clases de medios. En primer lugar, se encuentra un pequeño grupo de audiencia potencial alta, constituido por canales de televisión informativos y radioemisoras con audiencias mayores a 2.000.000 de seguidores. Un segundo grupo se caracteriza por una audiencia potencial media constituido por periódicos tradicionales con audiencias mayores a 500.000 seguidores. Finalmente, está el grupo de audiencia potencial baja, en el que cohabitan periódicos con foco temático específico y medios de pauta informativa alternativa con menos de 500.000 seguidores.

- También se pudo apreciar que la cobertura geográfica global de las informaciones resulta bastante completa al explorar el conjunto de medios seguidos (37), pero se pudo observar bastante variación entre la cobertura que hace cada medio.
- Las tendencias temáticas informativas parecen fuertemente orientadas a deportes y entretenimiento (más de un $50 \%$ en 30 medios), y marginalmente orientadas a sucesos policiales y judiciales (sólo en 3 medios superan el $10 \%$ de su producción informativa). Esta última tendencia parece marcar un cambio con respecto a la tradición chilena informativa, que siempre ha destinado gran cobertura a los sucesos policiales. Sin embargo, este cambio puede deberse a una tendencia editorial sólo aplicada a la información del público destinatario de Twitter, que etaria y socialmente está definido en un $65 \%$ como un público joven-adulto de entre 15 y 30 años aproximadamente.

- La centralización de la cobertura informativa parece mantenerse como una constante de la prensa chilena. En el ámbito nacional existen territorios de los que los medios no hablan (Magallanes y Aysén).

\section{Bibliografía}

Arriagada, A., Correa, T., Scherman, A. y Abarzúa, J. 2014. Diarios de Vida de las Audiencias Chilenas. Santiago: Universidad Diego Portales y Conicyt Pluralismo.

Azocar, A., Scherman, A., Arriagada, A., Pardo, J. y Becerra, A. 2010. Primer Estudio Nacional sobre Lectoría de Medios Escritos. Santiago: Universidad Diego Portales.

Baeza-Yates, R. y Pasi, G. 2011. "Special issue of The Journal of Information Retrieval on web mining for search". Information Retrieval, 14 (3): 213-214.

Baeza-Yates, R. y Sáez-Trumper, D. 2015. "Wisdom of the crowd or wisdom of a few? An Analysis of Users' Content Generation". Proceedings of the 26th ACM Conference on Hypertext \& Social Media. 69-74.

Baeza-Yates, R. 2009. "Tendencias en minería de datos de la Web". El profesional de la información, 18 (1): 5-10.

Cabalin, C. 2014. "Estudiantes conectados y movilizados: El uso de Facebook en las protestas estudiantiles en Chile". Comunicar, 43: 25-33.
Cárcamo-Ulloa, L., y Saez-Trumper, D. 2014. "Medios de Comunicación de Masas en Facebook. Comparativa de Chile y España”. XX Congreso Internacional de la Sociedad Española de Periodística (SEP). Barcelona, España.

2013. "¿Cambian las hegemonías periodísticas en las redes sociales? Prensa chilena en Facebook". Revista Nhengatu 1 (1).

Cardoso, G. 2014. "Movilización social y medios sociales". Vanguardia dossier, 50: 16-23.

ComScore. 2013. Futuro Digital Chile 2013. Recuperado de https://www.comscore.com/lat/Insights/Presentations and_Whitepapers/2013/2013_Chile_Digital_Future_in_Focus. (Consultado en febrero de 2016).

Consejo Nacional de Televisión (CNTV). 2014. Movilizaciones estudiantiles. Percepciones de los jóvenes. Santiago: Dpto de Estudios, Consejo Nacional de Televisión. Recuperado de http:// www.cntv.cl/movilizaciones-estudiantiles-percepcion-jovenes/ prontus_cntv/2014-04-04/154750.html (Consultado en Febrero 
de 2016)

Corrales. O. y Sandoval, J. 2005. "Medios de comunicación, pluralismo y libertad de expresión", Colección ideas, Cuadernos de trabajo Fundación Chile XXI. № 53.

Díaz-Nosty, B. 2013. La prensa en el nuevo ecosistema informativo " Que paren las rotativas!. Barcelona: Ariel.

García, R., Botella, F., y Marcos, M. C. 2010. "Hacia la arquitectura de la información 3.0: pasado, presente y futuro". El profesional de la información, 19 (4): 339-347.

Guzmán, J. y Poblete, B. 2013. "On-line relevant anomaly detection in the twitter stream: an efficient bursty keyword detection model". Proceedings of the ACM SIGKDD Workshop on Outlier Detection and Description, 31-39.

Halpern, D. 2014. Social TV en Chile: Hábitos y tendencias. Santiago, VTR - Facultad Comunicaciones UC - TrenDigital. Recuperado de http://www.iab.cl/social-chile-habitostendencias-facultad-comunicaciones-tren-digital-abril-2014/ (Consultado en Febrero de 2016).

Interactive Advertising Bureau (IAB). 2012. Uso de redes sociales en Chile. Recuperado de http://www.iab.cl/uso-deredes-sociales-en-chile-octubre-2012/ (Consultado en Febrero de 2016).

Joachims, T. 2002. Learning to classify text using support vector machines: Methods, theory and algorithms. Norwell: Kluwer Academic Publishers.

Kurth, M. 1993. "The limits and limitations of transaction log analysis", Library Hi Tech 11 (2): 98-104.

Maldonado, J., Peña-Araya, V. y Poblete, B. 2015. "Spatio and temporal characterization of chilean news events in social media". SIGIR 2015 Workshop on Temporal, Social and Spatially-aware Information Access (TAIA'15), Santiago, Chile.

Marcos, M. C., y González-Caro, C. 2010. "Comportamiento de los usuarios en la página de resultados de los buscadores. Un estudio basado en eye tracking". El profesional de la,19 (4): 348-358.

Masip, P., Díaz-Noci, J., Domingo, D., Micó-Sanz, J. L., y Salaverría, R. 2010. "Investigación internacional sobre ciberperiodismo: hipertexto, interactividad, multimedia y convergencia". El profesional de la información, 19 (6): 568- 576.

Morales, C., Soto, S. y Martínez, C. 2005. "Estado actual de la aplicación de la minería de datos a los sistemas de enseñanza basada en web". Actas del III Taller Nacional de Minería de Datos y Aprendizaje, TAMIDA2005, 49-56.

Newman, N. 2013. Reuters Institute Digital News Report 2013: Tracking the Future of News. Recuperado de https:// reutersinstitute.politics.ox.ac.uk/sites/default/files/Digital\%20 News\%20Report\%202013.pdf (Consultado en Febrero de 2016)

Pardo, N. 2012a. "Metáfora multimodal: Representación mediática del despojo". Forma y Función, 25 (2): 39-61. 2012b. Discurso en la Web: Pobreza en Youtube. Universidad Nacional de Colombia. Bogotá: Editorial Grafiweb.

Peters, T. 1993. "The history and development of transaction log analysis". Library Hi Tech, 42: 41-66.

Puente, S. y Grassau, D. 2009. "Informaciones regionales: calidad y presencia en la agenda noticiosa nacional". Cuadernos de Información, 25: 29-38.

Ramírez, J.D. 2009. "La concentración de la propiedad radial en Chile: las exigencias de nuevos paradigmas entre Globalidad y Localidad". Revista Redes.com, 5: 309-327.

Sadowsky, S. y Martínez, R. 2012. Lista de Frecuencias de Palabras del Castellano de Chile (Lifcach). Versión 2.0. Base de datos electrónica. http://sadowsky.cl/lifcach.html

Saez-Trumper, D., Castillo, C. y Lalmas, M. 2013. "Social media news communities: gatekeeping, coverage, and statement bias". Proceedings of the 22nd ACM international conference on Conference on information \& knowledge management. 1679-1684

Sáez-Trumper, D. 2011. "La información en Internet: Breve estado del arte para discutir el poder de los usuarios $\mathrm{v} / \mathrm{s}$ los medios tradicionales de comunicación en la red". Revista Austral Ciencias Sociales, 20: 71-79.

Sánchez, H. y Méndez, S. 2013. “¿Perfiles profesionales 2.0? Una aproximación a la correlación entre la demanda laboral y la formación universitaria". Estudios sobre el mensaje periodístico, 19: $981-993$

Stambuk, P. 1999. "El desequilibrio informativo en Chile: discriminación de la noticia regional en la prensa nacional". Revista Latina de Comunicación Social, 14. Recuperado de http://www. ull.es/publicaciones/latina/a1999c/133valpara.htm (Consultado en Febrero de 2016)

Tejedor-Calvo, S. 2010. "Web 2.0 en los ciberdiarios de América Latina, España y Portugal”. El profesional de la información, 19 (6): 610-619.

Vera, S. 2005. Concentración de la propiedad de los medios de comunicación en Chile y sus efectos en el pluralismo informativo (Memoria para optar al grado de Licenciado en Ciencias Jurídicas y Sociales). Valdivia: Universidad Austral de Chile.

Vergara, E., Garrido, C., Santibáñez, A. y Vera, P. 2012. "Inversión publicitaria y pluralismo informativo: Una aproximación comparada al caso de la prensa en Chile". Comunicación y Medios, 25: 57-70.

Vernier, M., Monceaux, L. y Daille, B. 2012. "Détection de la subjectivité et catégorisation de textes subjectifs par une approche mixte symbolique et statistique". Expérimentations et évaluations en fouille de textes: Un panorama des campagnes DEFT. Grouin, C. y Forest, D. (Eds.). Hermes-Lavoisier.

Yang, J. y Leskovec, J. 2011. "Patterns of temporal variation in online media". Proceedings of the fourth ACM international conference on Web search and data mining. 177-186. 
$\underline{\underline{202}}$ 